\title{
IIES
}

\section{UNDERSTANDING PENSIONS: COGNITIVE FUNCTION, NUMERICAL ABILITY AND RETIREMENT SAVING}

James Banks Zoë Oldfield 


\title{
Understanding pensions: Cognitive function, numerical ability and retirement saving
}

\author{
James Banks \\ University College London and Institute for Fiscal Studies \\ and \\ Zoë Oldfield \\ Institute for Fiscal Studies
}

First version: February 2004

Final version: February 2006

\begin{abstract}
Acknowledgements
We are grateful to Carl Emmerson and Gemma Tetlow for useful comments and to members of the IFS Pensions and Retirement Saving Consortium for funding this analysis and also for providing comments. The Consortium comprises HM Treasury, Department for Work and Pensions, Inland Revenue, Bank of England, Investment Management Association, The Actuarial Profession and the Association of British Insurers. Data from the English Longitudinal Study of Ageing (ELSA) were supplied by the ESRC Data Archive. ELSA was developed by researchers based at University College London, the Institute for Fiscal Studies and the National Centre for Social Research, with funding provided by the National Institute of Aging in the United States, and a consortium of UK government departments coordinated by the Office for National Statistics. Responsibility for interpretation of the data, as well as for any errors, is the authors' alone.
\end{abstract}




\section{Executive Summary}

As the degree to which individuals are expected to provide their own resources for retirement increases, there is a correspondingly increasing importance of individuals being able to understand the financial choices they face and to choose savings products, portfolios and contribution rates accordingly. In this paper we look at numerical ability and other dimensions of cognitive function in a sample of older adults in England and examine the extent to which these abilities are correlated with various measures of wealth and retirement saving outcomes. The key findings are:

a) Relatively large fractions of the population can be seen to have relatively low levels of financial numeracy and these numeracy levels decline systematically with age.

b) Numeracy levels are correlated with measures of retirement saving and investment portfolios, even when we control for other cognitive ability and education.

c) Numeracy is correlated with knowledge and understanding of pension arrangements, and with perceived financial security, even when we control for other cognitive ability, education and the level of overall retirement saving.

The lessons of our analysis are threefold, even though at this point we have only crosssectional data available on which to base our analysis. Firstly, it shows yet another dimension in which inequalities amongst older individuals are apparent. Second, the analysis suggests that in the short run there may be a role for targeting simple retirement planning information at low numeracy, low wealth, low education groups. Third, it suggests that a longer run policy goal might want to target numeracy levels more generally in order to reduce the fraction of the population with low basic skills. Whether such a policy would have knock on effects on to retirement planning arrangements, however, is a more difficult question to answer on the basis of the conditional correlations we present here. On this topic in particular, there is much further work to be done. 


\section{Introduction}

A succession of reforms to public pension provision in Britain over the last twenty years have led to a large and well-documented increase in the importance of individual provision for financial resources in retirement in the British welfare system. The majority of employees are now contracted out of the public second tier pension and have become members of some form of private pension instead. In addition, the income that most future retirees will receive from the state, either through the flat rate pension or through earnings related components, will fall relative to average earnings as a result of reforms reducing the generosity of state pension entitlements. As a result, future generations of pensioners are expected to have much larger fractions of their income coming from private, as opposed to public, sources.

Such a policy of transferring the responsibility for retirement saving from the state to the individual places increased demands on individual's ability to plan for their retirement or any unforeseen circumstances that may occur over their lifetimes, and on their willingness to implement these plans by foregoing consumption in working life. This has led to a heated debate about whether private saving will be adequate to finance suitable living standards in retirement for future cohorts. Regardless of what we think about the answer to this extremely complicated and multi-dimensional question, it seems natural to believe that the more straightforward the planning environment faced by individuals, the more likely it is that they will be able to deliver an appropriate degree of retirement income provision for themselves.

Apart from factors related to an individual's own expectations and preferences, there are four key dimensions that are important in individual's decision making processes. The first two are the simplicity and the stability of the policy environment in which individuals need to make choices. The third relates to the quantity and nature of information available to individuals about their retirement saving options. Finally, and interacting with all the previous factors, an individual's ability to process information and to form an understanding of an appropriate pension and savings plan - their 'financial' planning ability - is clearly going to be an important determinant of subsequent retirement outcomes. 
The UK system does not score highly on grounds of either simplicity or stability. Its pension system is particularly complex and successive governments have been unable to resist the temptation to make relatively major reforms to both the structure and the generosity of various components. Such reforms have tended to shy away from creating immediate losers they have tended to involve long (and complicated) transitional periods and additional components to the system (both of pension spending and tax relief) rather than remove existing elements. ${ }^{1}$

The extension of means testing further up the pensioner income distribution that is projected to occur over the next twenty years if current policy persists will only make things even more complicated, particularly in the low to middle lifetime income range where more and more people are likely to have the net return on their private saving reduced through withdrawal of other benefits. And although there has been recent policy debate on the importance of the provision of simple and understandable financial products (Pickering (2002), Sandler, (2002)), coupled with some momentum to provide better information on the value of pension entitlements and the need for retirement planning (DWP, 2003), there has only been partial movement in these domains (for example the introduction of the Sandler suite of lightly regulated stakeholder style products). Consequently, we are currently living in a time where an individuals ability to cut through the complexity and figure out their optimal retirement plans is probably more important than ever.

In this paper we will describe levels of basic numeracy amongst a cohort of people approaching their retirement in England. We show that relatively large proportions of the population are unable to carry out relatively straightforward financial calculations with accuracy. We then go on to look at how such numerical ability is correlated with various aspects of pension arrangements, focusing on both the level and form of saving, and also on peoples knowledge and expectations of their own retirement saving arrangements. The correlations are stark, and persist even when we control for other factors including education (years of schooling), levels of financial wealth, other family circumstances, and even other dimensions of cognitive functioning and abilities.

\footnotetext{
${ }^{1}$ Recent reforms and proposals have acknowledged this issue and consequently, as a result of the increasing complexity, simplification of pensions arrangements is now becoming an additional policy objective.
} 
We begin by defining our measure of numerical ability and showing some simple variation with age, gender and education as validation. In section three we discuss how numerical ability is associated with savings and wealth patterns. Finally, in section four we look specifically at the group approaching retirement - those aged 50 to 59 - and analyse the degree to which numerical ability is correlated with knowledge of pension arrangements, expectations of retirement income inadequacy and a more general measure of financial insecurity. Section five concludes.

\section{Measuring cognitive function for older individuals}

Our analysis will use the 2002 wave of the English Longitudinal Study of Ageing (ELSA) which is a survey of 11,400 individuals that provides a representative sample of the English population aged 50 and over on February 29 2002. The study contains a complete picture of financial circumstances (including savings and pension arrangements) as well as detailed information on health and socioeconomic factors (see Marmot et al (2003) for further details and description of the ELSA data). In addition, the questionnaire includes a module of questions designed to measure cognitive ability, within which a subset of questions allow us to evaluate respondent's numerical ability.

The cognitive measures in ELSA are designed to partition cognitive functioning into two broad domains, each with sub-components for which blocks of measures have been designed. The first domain relates to memory, with components comprising retrospective memory (recalling things from the past) and prospective memory (remembering to remember things in the future). The second domain, more relevant for our analysis, relates to executive functioning. In this domain the ELSA instrument comprises specific tasks (relating to verbal fluency, attention, visual search and mental speed) and a set of questions to identify numerical ability. Steel et al (2003) derive a global cognitive index from these items that takes a value between 1 and 60 and show that this index covaries in ways that one would expect. For example global cognitive function is lower in older age groups, and higher for individuals with more education or better health. 
In order to measure numerical ability, the ELSA questionnaire asked respondents up to five basic questions involving successively more complex numerical calculations. ${ }^{2}$ The six possible questions are presented in Box 1. Answers to all questions are entirely unprompted (i.e. respondents are not given a menu of possible answers to choose from). Each respondent initially receives questions q2, q3 and q4. If all of these are answered incorrectly the respondent receives question q1 and that is the end of their numeracy module. Otherwise the respondent receives question q5. If the respondent reports a correct answer to any (or all) of questions q3, q4 and q5, they receive the final and most difficult question q6 that requires an understanding of compound interest. Since more able individuals receive more questions in this design the number of questions answered correctly is a straightforward measure of numerical ability that can be derived simply from this module. This is the measure summarised by Steel et al (2003) in their initial descriptive analysis of the ELSA data.

\section{Box 1. Numeracy questions in 2002 ELSA questionnaire}

q1) If you buy a drink for 85 pence and pay with a one pound coin, how much change should you get?

q2) In a sale, a shop is selling all items at half price. Before the sale a sofa costs $£ 300$. How much will it cost in the sale?

q3) If the chance of getting a disease is 10 per cent, how many people out of 1,000 would be expect to get the disease?

q4) A second hand car dealer is selling a car for $£ 6,000$. This is two-thirds of what it cost new. How much did the car cost new?

q5) If 5 people all have the winning numbers in the lottery and the prize is $£ 2$ million, how much will each of them get?

q6) Let's say you have $£ 200$ in a savings account. The account earns ten per cent interest per year. How much will you have in the account at the end of two years?

\footnotetext{
${ }^{2}$ A similar study in the US - the Health and Retirment Study - delivered an experimental module of measures of financial numeracy to a subsample of their survey respondents, partly with the aim of comparing to the ELSA measures, in their 2004 wave. Although the questions are not strictly comparable, a descriptive analysis of this US data and how it correlates with retirement saving arrangements is presented in Lusardi and Mitchell (2005)
} 
The numerical ability measure we derive from the ELSA data is instead designed to place individuals into one of four groups according to their broad numerical ability. This has the advantage of allowing us to choose groups that have some prevalence in the population since a simple counting of correct answers does not take into account the relative difficulty of the questions and furthermore may lead to some clusters where there are many observations, with relatively few individuals at the extremes. Hence for our analysis we choose to define numerical ability in four broad groups according to which of the questions were correctly answered. This coding is indicated in Table 2.1.

Figure 2.1 shows how these four categories of numerical ability co-vary with age and gender. As with the more aggregated analysis in Steel et al (2003) who simply report the average number of correct answers by group, numerical ability as defined by these measures is greater for men than for women, and greater for younger individuals than for their older counterparts. Even for the group aged 50-54 there are a substantial proportion of respondents in the bottom category and relatively few who correctly answered all questions, particularly for women.

Table 2.1: Construction of broad cognitive function categories

Classification Response to questions

Proportion of sample
Group I
Either: q2, q3, q4 all incorrect
$16.24 \%$
Or: $\quad$ q2 correct; q3, q4, q5 all incorrect
Group II
At least one of q2, q3, q4, q5 incorrect; q6 incorrect
$46.46 \%$
Group III
$\mathrm{q} 2, \mathrm{q} 3, \mathrm{q} 4, \mathrm{q} 5$ correct; q6 incorrect
$26.08 \%$
Group IV
$\mathrm{q} 2, \mathrm{q} 3, \mathrm{q} 4, \mathrm{q} 5, \mathrm{q} 6$ correct
$11.22 \%$ 
Figure 2.1 Broad numeracy score, by age and gender

Men

Women

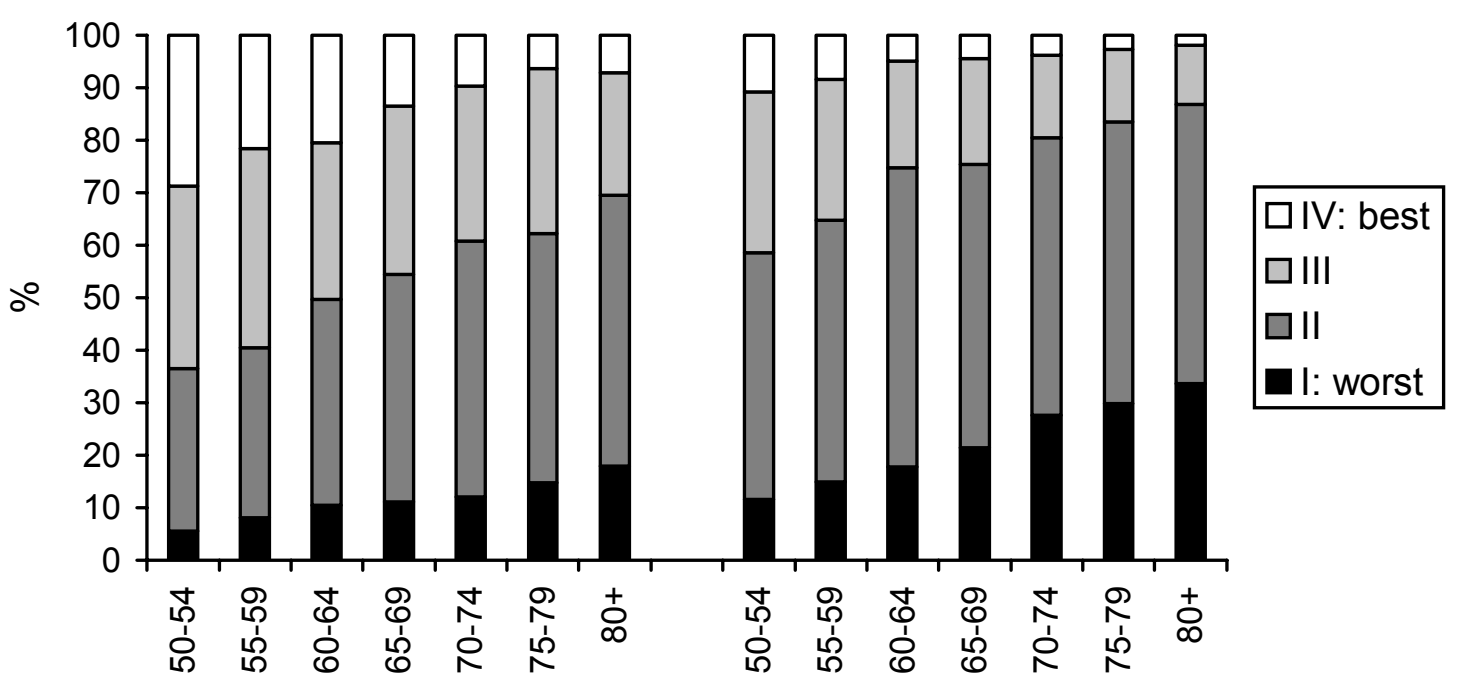

In Figure $2.2 \mathrm{a}$ and $2.2 \mathrm{~b}$ we decompose this analysis further to split by education, although compared to the previous figure we aggregate to three broad age groups for simplicity. The association between numerical ability and education is clearly evident in the data. As one would expect - groups with higher education have higher numerical ability. Notably however, the age pattern in the 'decline' in numerical ability is much stronger for the more educated groups, both for men and women, particularly at the top end. Thus the differences across education groups, whilst still present, are less marked in the oldest members of the ELSA sample in comparison to the 50-59 year olds. It should be said, however, that our data is cross-sectional in nature, and the presence of either differential mortality (the rich and able living longer than their poor and less able counterparts) and the presence of cohort differences in numeracy will mean that these correlations may not be the true age profiles. 
Figure 2.2a Broad numeracy score, by age and education: Men

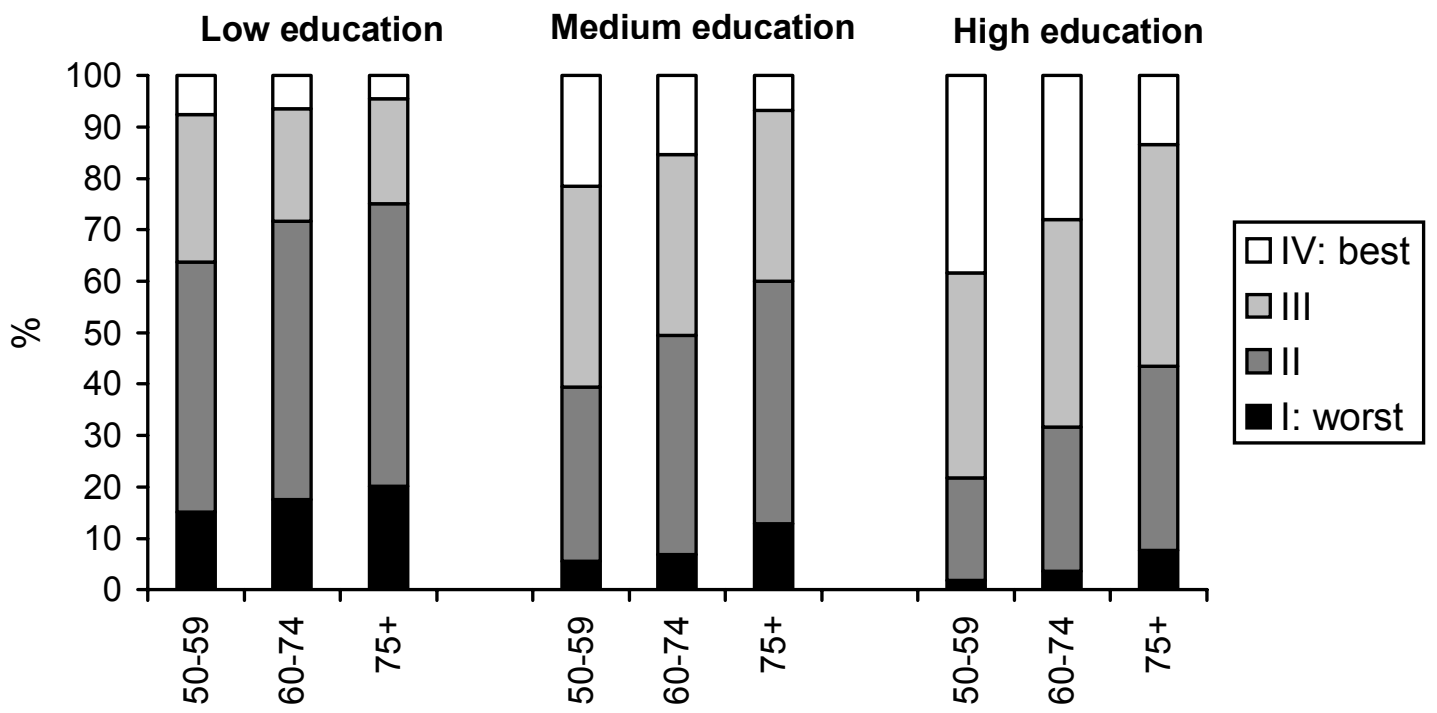

Figure 2.2b Broad numeracy score, by age and education: Women

Low education Medium education High education

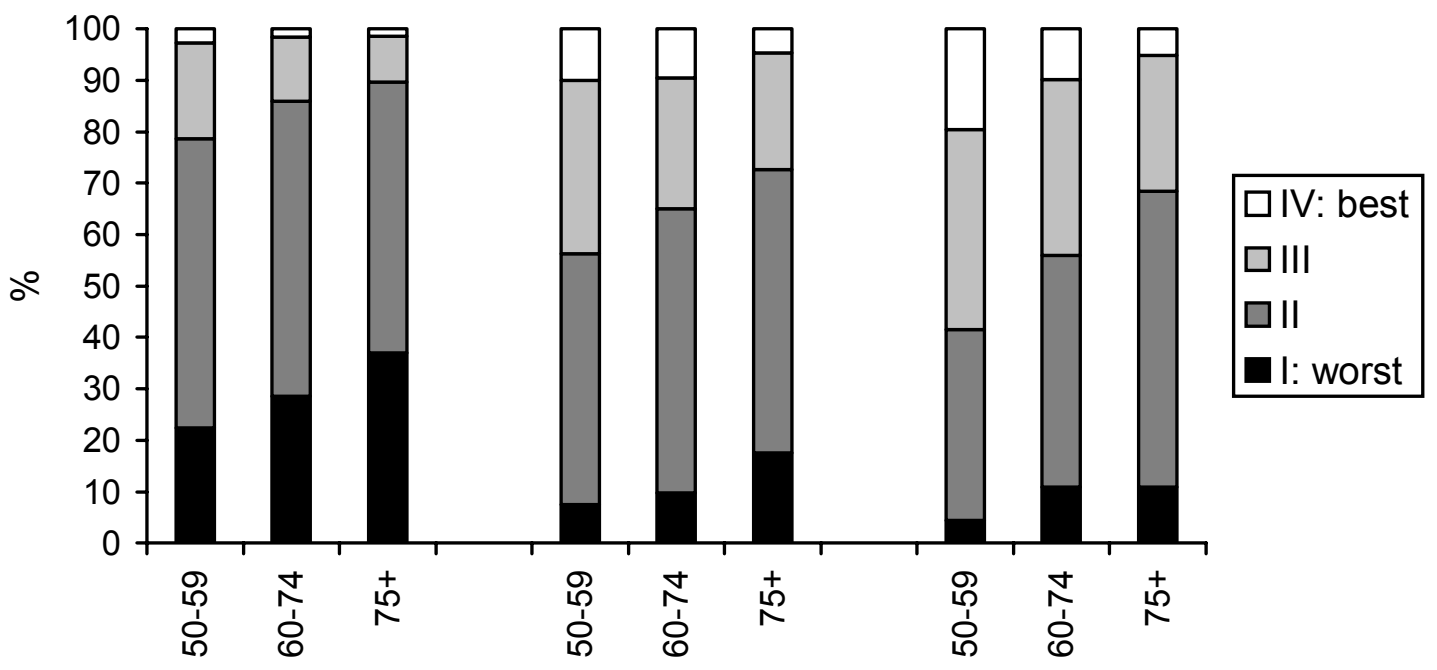

Whilst the true age profile will only be revealed in longitudinal data, it is possible to speculate on the sign of such biases. Differential mortality would reduce the extent to which we observe a decline with age in our sample and, to the extent that compound and simple interest was more likely to be taught to the older members of our sample, cohort differences in the nature of education would work the same way. In these circumstances the age related decline observed in figures $2.1,2.2 \mathrm{a}$ and $2.2 \mathrm{~b}$ may even be an 
underestimate. On the other hand, cohort effects in the proportion of individuals with different levels of education (if this extra education led to higher levels of numeracy) would work the other way.

One cohort effect is undoubtedly important - the difference between men and women in the cohorts currently aged 50 and over taken together (i.e. the whole ELSA sample) is unlikely to be indicative of similar differences for future cohorts of such individuals. Current and future generations of working age women have very similar educational and labour market circumstances to their male counterparts and, if such circumstances lead to higher levels of numeracy, one might expect future generations of older women to be more comparable to men.

Finally, three other points are worth noting about the classification of numeracy we adopt in this paper. Firstly, our classification mirrors the patterns shown when using the question count definition adopted by Steel et al (2003) although the latter analysis only looks at age, gender and education one dimension at a time. Secondly, for all the analysis that follows we have experimented with categorising the population into three groups (aggregating categories III and IV) and into five groups (disaggregating the largest group - group II - into two sub-groups according the number of incorrect questions) and our conclusions are unaffected. Finally, and important to bear in mind when interpreting the multivariate analysis in sections four and five, Figures 2.2a and $2.2 \mathrm{~b}$ show that even within education groups there is a reasonably good distribution of the population across the four numerical abilities. This is particularly true for the 50-59 year olds on who the analysis in the latter sections will focus. There are individuals with low numerical ability in the highest education groups and individuals with high cognitive ability in the low and medium education groups. The presence of such variation is important if we are to look at the separate effects of the two factors on pension understanding and financial insecurity. 


\section{Correlations between numeracy and financial asset holding}

The previous section shows that there is a large amount of variation in numerical ability amongst the population aged 50 and over. In this section will investigate the extent to which this is correlated with both the amount of financial wealth held by individuals and the form in which this wealth is held. Of course, wealth is a stock variable - one which represents the sum of past saving decisions - and one might argue that current cognitive ability is only one part of the story. Maybe cognitive function in earlier life when the wealth accumulation decisions were being made is the more relevant factor.

Unfortunately we do not have such a measure in our data. Nevertheless, if cognitive function does not decline until age 50 our measure of ability will be the correct one. In addition, since we use a relative measure of wealth - the individual's position in the wealth distribution rather than the number of thousands of pounds they have accumulated - our analysis should also be more robust to any changes in functioning that do occur before age 50 .

\subsection{The correlation between numeracy and wealth}

Figures 3.1a and 3.1b show the correlation between numeracy and financial wealth for 50-59 and 60-74 year olds respectively. Financial wealth is defined as the value of all non-pension financial assets less any outstanding debts (not including mortgage debts). In each (age-group specific) wealth quintile, the figures show the percentage of individuals

found in the four numeracy groups. ${ }^{3}$ For both age groups and for men and women there is a clear correlation between numeracy and wealth. Amongst 50-59 year old men, those with the highest numerical ability are over 2.5 times more likely to be found in the highest wealth quintile while those with the worst numerical ability are over 6 times more likely to be found in the poorest wealth quintile. Similar (although even steeper) gradients are found for women aged 50-59 and for both men and women aged 60-74.

\footnotetext{
${ }^{3}$ Financial wealth is defined at the benefit unit level (either a couple or single plus any dependent children they have) and numeracy is defined at the individual level. The issue of joint decision making is discussed in Table 3.2b below.
} 
Figure 3.1a Broad numeracy score, by age and financial wealth, ages 50-59

Men

Women

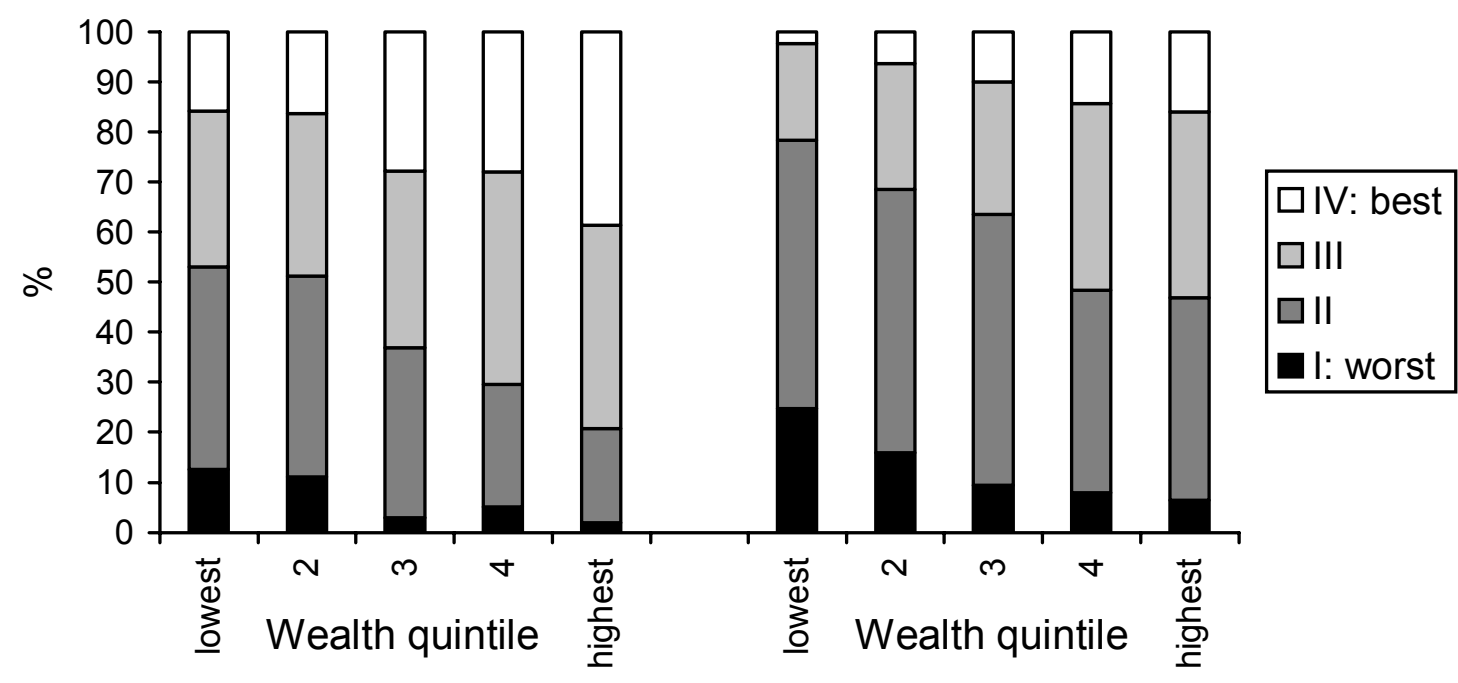

Figure 3.1b Broad numeracy score by age and financial wealth, ages 60-74

Men

Women

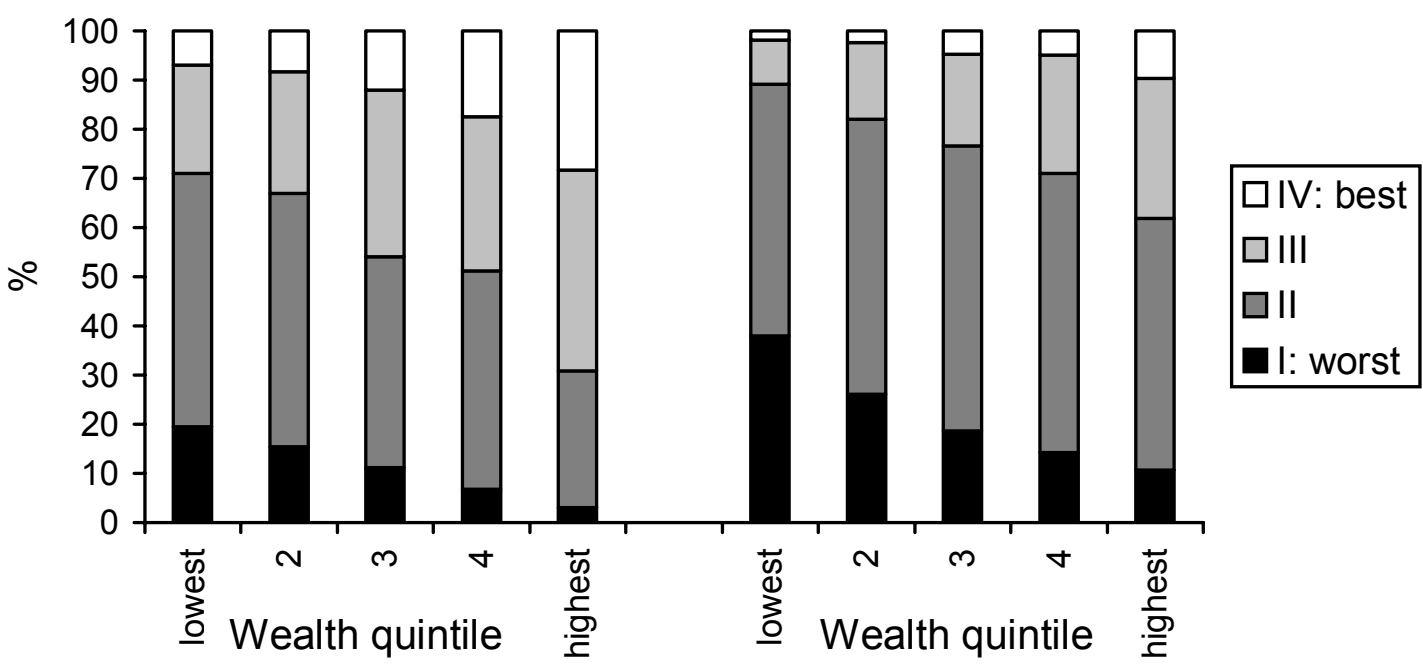

We showed in section 2 that there is a strong (but certainly not perfect) correlation between current numeracy and education. One question that might be asked is whether numeracy is important over and above education and other dimensions of cognitive ability when it comes to the relationship with wealth. Is there something about numeracy 
which helps people make financial decisions where more general education or ability does not necessarily help? We can try and disentangle these effects using a regression framework. Table 3.1a shows the results of multivariate analysis of the effects of financial wealth on numeracy group and education (and including other controls) that uses a Least Absolute Deviation estimator to reduce the sensitivity of estimates to the highly skewed nature of the distribution of wealth. The base group is defined as individuals with low numeracy and low education where low education is defined as having no academic qualifications, medium education is defined as having o-levels or equivalent and high education is defined as having a- 'levels (or equivalent) or higher.

To try and control fully for other levels of ability in addition to education we include measures derived from the other cognitive functioning tests in the ELSA data. These are not central to the paper so they are not discussed in detail but they cover executive function - the ability to do simple cognitive tasks - and memory - both prospective and retrospective. ${ }^{4}$ Scores in the sub components of each dimension are used to construct an index for that dimension and each of these indices are then entered quadratically in all the regressions that follow. These measures ought to capture at least some of the unobserved ability (and hence earnings power) within education groups and help us isolate the pure numeracy effect in the tables that follow.

\footnotetext{
${ }^{4}$ More specifically, executive functioning is measured by two tests - naming as many animals as you can in a one minute period, and a letter cancellation test where an individual has to work their way through a sheet of single letters crossing out all the P's and W's and where they are scored on both speed and accuracy. Memory is measured by two tasks - remembering a word list (both immediately and after a short delay) and successfully carrying out an instruction given to the respondent earlier on in the interview. See Steel et al (2003) for futher details of the measures and how the indices are defined.
} 
Table 3.1a. The correlation of financial wealth with numeracy and education

\begin{tabular}{|c|c|c|c|c|}
\hline & \multicolumn{2}{|c|}{ Aged 50-59 } & \multicolumn{2}{|c|}{ Aged $60-74$} \\
\hline & coefficient & t-ratio & coefficient & t-ratio \\
\hline Group II & 927 & 0.58 & 2,373 & 2.50 \\
\hline Group III & 7,071 & 4.08 & 7,470 & 6.60 \\
\hline Group IV & 11,450 & 5.81 & 19,434 & 13.49 \\
\hline Medium education & 3,257 & 2.96 & 7,205 & 8.67 \\
\hline High education & 12,283 & 11.35 & 23,005 & 28.13 \\
\hline Executive function & -615 & -1.54 & -169 & -0.59 \\
\hline Exec func ${ }^{2}$ & 46 & 2.70 & 22 & 1.69 \\
\hline Memory & -803 & -1.85 & -421 & -1.44 \\
\hline Memory ${ }^{2}$ & 39 & 3.10 & 27 & 2.93 \\
\hline Female & 2,460 & 2.81 & 2,395 & 3.62 \\
\hline Couple & 7,380 & 7.26 & 7,325 & 10.60 \\
\hline Sample size & 4,047 & & 4,737 & \\
\hline
\end{tabular}

Note: Estimates using weighted quantile (median) regression. All specifications also include full age dummies. Medium education is defined as $\mathrm{A}$ levels or equivalent post compulsory education. High education is defined as degree or equivalent,

The first two columns of Table 3.1a show the results for those aged 50-59 and the second two show the results for those aged 60-74. As would be expected, having medium or high education is associated with increased wealth compared to having no qualifications but even after controlling for education, being in the highest or second to highest numeracy group is still (significantly) associated with higher financial wealth. Indeed the gradient by numerical ability, even conditional on education, is equally strong as the gradient by education - the median individual with either high numerical ability or high education has accrued, on average and other things being equal, around $£ 12,000$ more financial wealth by their fifties than the base group. An individual with both high numerical ability and high education would on average have accrued around $£ 24,000$ more financial wealth by this time.

The association of wealth with cognitive function over and above education is particularly clear for those aged 60-74 in comparison to the younger group. It might be tempting to draw a number of conclusions from this, such as statements about the 'types' of people for whom cognitive function declines the most or even the 'causes' of cognitive decline or wealth decumulation. However, two important caveats must be borne in mind. Firstly the fact that this is simply a cross-section of individuals makes such arguments 
wholly unsubstantiated. Secondly, the comparison of wealth between 50-59 year olds and 60-74 year olds is confounded by the fact that the younger group are yet to retire and their pension wealth will not be captured in our definition of financial wealth. In contrast, given that a large fraction of the older group will have retired any lump sums they may have received from their private pensions or any pension wealth they have received but are yet to annuitise will be measured as financial wealth. Whilst this is also true for the 50-59 age group the likelihood of individuals already having received such sums is, of course, lower.

Finally in this section, we address the issue that individuals within benefit units often act jointly and, as such, responsibility for saving and wealth accumulation decisions may be shared with more (or less) numerically able spouses. Since the ELSA survey delivers cognitive tests to both individuals in the couple we are able to construct measures of numeracy and education that reflect the maximum of the values for the two individuals in the Benefit Unit - that is they reflect the abilities and education of the more able of the two members of the couple. Table $3.1 \mathrm{~b}$ repeats the analysis above with these Benefit Unit levels of numeracy and ability in place of the individual measures used in Table 3.1a above. The broad conclusions are all unchanged although the wealth gradient by numeracy level is now somewhat different to that observed between the top and the bottom of the education distribution.

Table 3.1b. The correlation of financial wealth with numeracy and education

\begin{tabular}{|c|c|c|c|c|}
\hline & \multicolumn{2}{|c|}{ Aged 50-59 } & \multicolumn{2}{|c|}{ Aged $60-74$} \\
\hline & coefficient & $t$-ratio & coefficient & t-ratio \\
\hline BU Group II & -796 & -0.42 & 796 & 0.94 \\
\hline BU Group III & 5,192 & 2.59 & 6,789 & 7.03 \\
\hline BU Group IV & 13,376 & 6.21 & 16,056 & 14.02 \\
\hline BU Medium educ & 2,080 & 1.79 & 5,195 & 7.85 \\
\hline BU High educ & 9,147 & 8.19 & 22,849 & 35.40 \\
\hline Executive function & -946 & -2.23 & -91 & -0.40 \\
\hline Exec func ${ }^{2}$ & 57 & 3.41 & 13 & 1.22 \\
\hline Memory & -781 & -1.62 & -349 & -1.43 \\
\hline Memory ${ }^{2}$ & 38 & 2.87 & 25 & 3.33 \\
\hline Female & $-1,183$ & -0.74 & -173 & -0.22 \\
\hline Couple & 3,655 & 2.68 & 3,156 & 4.28 \\
\hline Sample size & 3,108 & & 3,076 & \\
\hline
\end{tabular}

Note: Estimates using weighted quantile (median) regression. All specifications also include full age dummies. Sample is one observation per benefit unit only. 


\subsection{The Correlation between numeracy and portfolio composition}

Total financial wealth is composed of different types of assets. Some financial assets are fairly universally held and are relatively simple in the way they operate. Others are more complex and may also be more risky. Are less numerate individuals less likely to hold these more complex types of asset? To answer this question, we look at the effect of numeracy on the probability of owning different types of assets (savings accounts, shares and private pensions) after controlling for education and financial wealth and other relevant individual characteristics. The results are shown in Tables 3.2a (ages 50-59) and $3.2 \mathrm{~b}$ (ages 60-74). The results show that the marginal effect of increased numeracy on the probability of owning a savings account is small for both age groups. This is to be expected as the majority (around 90\%) of individuals aged 50-59 and 60-74 own a savings account.

The relationship between numeracy and share ownership is much stronger for both age groups but particularly strong for the younger group. Even after controlling for the level of wealth, education and other cognitive abilities, compared to the least numerate group the most numerate group are 15 percentage points more likely to own shares and even numeracy group II are around 7 percentage points more likely to own shares. The marginal effects of numeracy on the probability of owning shares are smaller (but still statistically significant) for the older (60-74 year old) group. ${ }^{5}$

The final two columns show the marginal effects of the various characteristics on the probability of owning a private pension (defined as currently contributing to a private pension or ever having contributed to a private pension). Those aged 50-59 in the most numerate group, are around 10 percentage points pension (and in the 60-74 year old group, around 11 percentage points) more likely to own a private pension than those in the least numerate group and there is a significant positive marginal effect of being in group II or III compared to the least numerate group in both age groups.

\footnotetext{
${ }^{5}$ Whilst we do not present the models at the benefit unit level using the most numerate adults cognitive scores our conclusions are unchanged if we run our analysis on such a basis (as in Table $3.1 \mathrm{~b}$ above).
} 
Table 3.2a.Asset ownership and numeracy, ages 50-59

\begin{tabular}{|c|c|c|c|c|c|c|}
\hline & $\begin{array}{c}\text { Savings } \\
\text { Marg. } \\
\text { effect }\end{array}$ & unt & $\begin{array}{c}\text { Sh } \\
\text { Marg. } \\
\text { effect }\end{array}$ & $z$ & $\begin{array}{c}\text { Private } \\
\text { Marg. } \\
\text { effect }\end{array}$ & on \\
\hline Group II & 0.008 & 0.77 & 0.069 & 1.87 & 0.062 & 2.82 \\
\hline Group III & 0.001 & 0.05 & 0.121 & 3.09 & 0.076 & 3.17 \\
\hline Group IV & 0.013 & 0.95 & 0.153 & 3.45 & 0.105 & 3.86 \\
\hline Medium education & 0.014 & 1.73 & 0.025 & 1.13 & 0.075 & 4.89 \\
\hline High education & 0.008 & 1.00 & 0.047 & 2.12 & 0.159 & 9.80 \\
\hline wealth quintile 2 & 0.060 & 10.95 & 0.193 & 6.09 & 0.088 & 4.99 \\
\hline wealth quintile 3 & 0.072 & 11.90 & 0.373 & 12.95 & 0.130 & 7.63 \\
\hline wealth quintile 4 & 0.083 & 12.72 & 0.487 & 17.84 & 0.142 & 8.40 \\
\hline wealth quintile 5 & 0.094 & 12.03 & 0.616 & 23.15 & 0.133 & 7.42 \\
\hline Executive function & 0.055 & 2.00 & -0.002 & -0.29 & -0.004 & -0.66 \\
\hline Exec func ${ }^{2}$ & -0.000 & -1.29 & 0.000 & 0.27 & 0.000 & 1.37 \\
\hline Memory & -0.001 & -0.47 & 0.019 & 1.91 & -0.003 & -0.53 \\
\hline Memory $^{2}$ & 0.000 & 0.79 & -0.000 & -1.02 & 0.000 & 0.99 \\
\hline Female & -0.001 & -0.21 & -0.005 & -0.31 & -0.249 & -17.68 \\
\hline Couple & 0.052 & 6.12 & 0.130 & 6.01 & 0.005 & 0.32 \\
\hline Mean of dep var & 0.90 & & 0.40 & & 0.76 & \\
\hline
\end{tabular}

Note: Probit analysis. Age dummies also included. Sample size $=4047$

Table 3.2b. Asset ownership and numeracy, ages 60-74

\begin{tabular}{|c|c|c|c|c|c|c|}
\hline & $\begin{array}{c}\text { Savings } \\
\text { Marg. } \\
\text { effect }\end{array}$ & $\begin{array}{l}\text { unt } \\
z\end{array}$ & $\begin{array}{c}\text { Sh } \\
\text { Marg. } \\
\text { effect }\end{array}$ & $z$ & $\begin{array}{c}\text { Private } \\
\text { Marg. } \\
\text { effect }\end{array}$ & on \\
\hline Group II & 0.014 & 2.04 & 0.067 & 2.73 & 0.081 & 3.73 \\
\hline Group III & 0.028 & 3.59 & 0.078 & 2.72 & 0.107 & 4.19 \\
\hline Group IV & 0.022 & 2.19 & 0.034 & 0.99 & 0.114 & 3.28 \\
\hline Medium education & -0.001 & -0.19 & 0.042 & 2.20 & 0.083 & 4.32 \\
\hline High education & 0.003 & 0.34 & 0.084 & 4.41 & 0.160 & 8.11 \\
\hline wealth quintile 2 & 0.062 & 14.96 & 0.171 & 4.84 & 0.078 & 3.44 \\
\hline wealth quintile 3 & 0.059 & 14.05 & 0.410 & 11.93 & 0.175 & 8.01 \\
\hline wealth quintile 4 & 0.073 & 14.10 & 0.537 & 16.18 & 0.188 & 8.52 \\
\hline wealth quintile 5 & 0.069 & 12.99 & 0.693 & 21.57 & 0.207 & 8.89 \\
\hline Executive function & 0.003 & 1.37 & -0.004 & -0.61 & 0.004 & 0.58 \\
\hline Exec func ${ }^{2}$ & -0.000 & -0.81 & 0.000 & 1.06 & 0.000 & 0.10 \\
\hline Memory & 0.000 & 0.23 & 0.012 & 1.64 & 0.001 & 0.08 \\
\hline Memory $^{2}$ & 0.000 & 0.06 & -0.000 & -1.39 & 0.000 & 0.55 \\
\hline Female & 0.003 & 0.47 & 0.015 & 0.97 & -0.357 & -22.89 \\
\hline Couple & 0.018 & 3.11 & 0.046 & 2.80 & -0.028 & -1.68 \\
\hline Mean of dep var & 0.92 & & 0.32 & & 0.64 & \\
\hline
\end{tabular}

Note: Probit analysis. Age dummies also included. Sample size $=4737$ 
The results in Tables 3.1 and 3.2 certainly do not show unequivocally that being less numerate causes people to save less and to be less likely to hold risky assets. Indeed the direction of causality could run in the reverse direction - holding more wealth with a larger portion in more complex types of assets could allow people to learn and become more numerate. To begin to answer questions about causality would require more data and more rigorous analysis.

Nor necessarily is it a policy problem that less numerate individuals have lower wealth and are less likely to hold riskier assets or pensions. Indeed there may be good reasons for such differences. There could be some factor that we have not controlled for which captures need or desire for savings (for example, life expectancy) If this factor is correlated with numeracy, it would be optimal for less numerate individuals to save less. Alternatively more forward-looking people might both save more and also be more likely to choose to undertake more education, thus generating the same correlation by a different mechanism.

It should also be mentioned that holding risky assets is not necessarily optimal for all individuals as each will have a different degree of risk aversion and there may be fixed costs associated with holding more risky assets (such as transaction or management charges) that mean they would not be 'optimally' chosen by low wealth individuals, particularly if any information costs associated with investing in the asset are, in some sense, higher for those with low numeracy.

The above issues relate back to the difficult question of the extent to which people are saving optimally for their retirement. Perhaps the most important thing for the more limited questions we are looking at here is the simpler question of whether individuals are making fully informed choices and making their financial decisions based on information that they fully understand. In the next section, in order to try to understand the extent to which individuals do understand the financial products that they own, we look at the correlation between numeracy and financial knowledge, and in particular pension arrangements. 


\section{Correlations between numeracy and financial knowledge}

Information regarding pension arrangements and financial circumstances is collected in great detail in ELSA. Individuals are asked about the type of pension that they have and various details about how the pension plan operates. In addition they are asked about their expectations for future retirement income, and whether they feel that they have had 'enough' information from their pension plan. As such, there is a huge potential set of questions on which to draw when considering how much respondents know about their pension arrangements. It is somewhat inevitable that individuals will not know the answer to every one of these questions. In fact, not knowing the answer is an interesting outcome in itself as it provides information on how much people understand their own pension arrangements. This is particularly the case if we can identify groups who are more likely to know or not know their pension details. In light of this, "don't know" was explicitly listed as a possible response category on the cards that were shown to ELSA respondents in order to signal that this is a legitimate answer. In the next two sections we use these responses to try and understand what types of people are more likely to understand their pension arrangements and in particular whether numeracy has a part to play.

The first two columns in Table 4.1 report the results of a probit regression indicating whether the respondent knows the accrual rate in their pension plan. More precisely, the dependent variable is a dichotomous indicator variable that takes the value one when the individual reports a positive value to the following question: "What fraction will be added to your final pension for each years service?" Since the question relates to Defined Benefit employer pensions, the regression is carried out only on those with this sort of pension and we include both men and women aged 50-59. Overall, 58\% of individuals know the answer to this question. ${ }^{6}$ As well as numerical ability, we also control for education and financial wealth quintile, gender, whether the individual has a partner and age (although since we have a fairly homogeneous age group a single age dummy to capture whether the individual is $50-54$ or 55-59 is sufficient). The marginal

\footnotetext{
${ }^{6}$ Of course some individuals may report an incorrect answer but throughout this section we will be leaving this issue to one side. In principle, since ELSA respondents are asked the name of their pension plan, it would be possible to link respondents to their true plan details in order to assess whether answers were correct or not.
} 
effects for numerical ability show that compared to the least numerate group, the most numerate group are almost 35 percentage points more likely to know their pension accrual rate. Note that this is even after controlling for education, cognitive function and an individuals position in the financial wealth distribution, which also have the expected positive effects. Although the point estimates suggest that Groups II and III are also more likely to know their accrual rate than those in Group I these differences are less precisely estimated.

Table 4.1 DB Pension knowledge, aged 50-59

\begin{tabular}{|c|c|c|c|c|}
\hline & \multicolumn{4}{|c|}{ Whether respondent knows.... } \\
\hline & \multicolumn{2}{|c|}{ Accrual rate } & \multicolumn{2}{|c|}{ Expected pension income } \\
\hline & Mar. eff & $z$ & Mar. eff & $z$ \\
\hline Group II & 0.166 & 1.60 & 0.117 & 1.59 \\
\hline Group III & 0.196 & 1.88 & 0.190 & 2.52 \\
\hline Group IV & 0.347 & 3.47 & 0.200 & 2.66 \\
\hline Medium education & 0.164 & 2.90 & 0.007 & 0.16 \\
\hline High education & 0.209 & 3.89 & 0.011 & 0.26 \\
\hline $\mathrm{F}$ wealth quintile 2 & 0.095 & 1.38 & 0.027 & 0.50 \\
\hline $\mathrm{F}$ wealth quintile 3 & 0.151 & 2.55 & 0.058 & 1.20 \\
\hline$F$ wealth quintile 4 & 0.163 & 2.82 & 0.076 & 1.62 \\
\hline $\mathrm{F}$ wealth quintile 5 & 0.176 & 3.02 & 0.129 & 2.69 \\
\hline Executive function & -0.003 & -0.17 & 0.008 & 0.55 \\
\hline Exec func ${ }^{2}$ & 0.000 & 0.35 & -0.000 & -0.29 \\
\hline Memory & -0.029 & -1.26 & -0.033 & -1.80 \\
\hline Memory ${ }^{2}$ & 0.001 & 1.31 & 0.001 & 2.33 \\
\hline Female & -0.128 & -3.41 & -0.144 & -4.47 \\
\hline Couple & 0.061 & 1.21 & 0.026 & 0.63 \\
\hline Mean of dep var & 0.58 & & 0.70 & \\
\hline
\end{tabular}

Probit analysis. Age dummies also included

Sample: those currently contributing to an occupational pension, $N=1,103$

A second question that is asked of ELSA respondents with a defined benefit employer pension scheme is "How much do you expect to get from this pension when you retire?" The second two columns in Table 4.1 show the results of a probit regression where the dependent variable is equal to one if the respondent reports a value at this question. Overall, 70\% of individuals report some knowledge of expected pension income but the results show that those who are in numerical ability groups III are 19 
percentage points more likely and Group IV are 20 percentage points more likely to report a value than those who are in the least numerate group. Those in the Group II are not significantly more likely to report a value at this question than the least numerate group. Women are significantly less likely than men to know the answers to both the questions in Table 4.1.

Individuals are also asked about indexation of their pension scheme which will be key to the value of the income it will provide in retirement. More specifically they are asked "Will your pension go up by more than prices after retirement?". Table 4.2 shows the results of a probit regression that uses answers to this question as the dependent variable. In the first two columns, the dependent variable takes the value one if individuals answer either yes or no (i.e. if they do not report “don't know"). The results do not suggest that the more numerate are significantly more likely to report an answer to this question than the less numerate.

Table 4.2 Indexation of DB pension

\begin{tabular}{|c|c|c|c|c|}
\hline & \multicolumn{4}{|c|}{ Will your pension go up more than prices after retirement? } \\
\hline & \multicolumn{2}{|c|}{ "Knows" the answer } & \multicolumn{2}{|c|}{ Reports "no" } \\
\hline & Mar. eff & $z$ & Mar. eff & $Z$ \\
\hline Group II & 0.022 & 0.26 & 0.092 & 0.89 \\
\hline Group III & 0.067 & 0.82 & 0.132 & 1.27 \\
\hline Group IV & 0.105 & 1.25 & 0.167 & 1.54 \\
\hline Medium education & 0.054 & 1.11 & -0.042 & -0.71 \\
\hline High education & 0.053 & 1.15 & 0.023 & 0.43 \\
\hline F wealth quintile 2 & 0.050 & 0.87 & -0.030 & -0.43 \\
\hline F wealth quintile 3 & 0.089 & 1.78 & 0.022 & 0.36 \\
\hline F wealth quintile 4 & 0.034 & 0.69 & 0.020 & 0.33 \\
\hline F wealth quintile 5 & 0.111 & 2.24 & 0.056 & 0.94 \\
\hline Executive function & -0.025 & -1.30 & -0.005 & -0.25 \\
\hline Exec func ${ }^{2}$ & 0.001 & 0.90 & 0.000 & 0.19 \\
\hline Memory & 0.100 & 0.51 & 0.052 & 1.80 \\
\hline Memory $^{2}$ & -0.000 & -0.48 & -0.001 & -1.63 \\
\hline Female & -0.151 & -4.54 & -0.105 & -2.78 \\
\hline Couple & -0.033 & -0.75 & 0.050 & -0.98 \\
\hline Mean of dep var: & 0.73 & & 0.49 & \\
\hline
\end{tabular}

Probit model. Age dummies also included

Sample: those aged 50-59 with an defined benefit pension: $N=1,103$ 
However, of the individuals who report an answer to the indexation question, over $30 \%$ report that their pension will go up by more than prices whereas administrative data suggests that only a minority of pension plans are indexed by more than prices. The fact that so many individuals believe that their pension will go up by more than prices might indicate another area where there is lack of understanding about pensions. In the second two columns in Table 4.2 we therefore take a different approach. In this regression, the dependent variable takes the value one if an individual does not know the answer or they report that their pension will go up by more than prices. While this will overestimate the number of people who do not understand the way their pension works, it may reflect knowledge more accurately than assuming that individuals report the correct answer to this question. Using this alternative approach around half of individuals understand the way their pension scheme is indexed and numerical ability groups II, III and IV are more likely to understand indexation of their pension than the least numerate group although once again these differences are not statistically significant at the $95 \%$ level. Indeed, in general there are fewer statistically significant factors associated with this particular definition of a lack of pension knowledge. Instead, the lack of knowledge regarding indexation seems to be relatively constant across the distribution of wealth, education and numeracy.

So far we have used self-reported answers to questions about administrative details of pension schemes as a proxy for how well individuals understand their schemes. We now turn to an alternative question that directly asks individuals about the amount of information they have received about their pension: "Do you feel that you have received enough information about your expected pension at retirement?". The results of a probit regression where this variable (yes $=1$ ) is the dependent variable are reported in Table 4.3. Overall, around $70 \%$ of individuals say they feel they had received enough information about their current pension. The results show that only the most numerate are significantly more likely to feel that they had enough information compared to the least numerate group. 
Table 4.3 Feels had enough information about current pension

\begin{tabular}{l|cc}
\hline & \multicolumn{2}{|c}{$\begin{array}{c}\text { Currently contributing } \\
\text { Mar. eff }\end{array}$} \\
\hline Group II & 0.060 & 1.25 \\
Group III & 0.066 & 1.31 \\
Group IV & 0.158 & 3.16 \\
Medium education & -0.035 & -1.21 \\
High education & -0.014 & -0.50 \\
F wealth quintile 2 & -0.000 & -0.01 \\
F wealth quintile 3 & 0.090 & 2.89 \\
F wealth quintile 4 & 0.101 & 3.32 \\
F wealth quintile 5 & 0.103 & 3.28 \\
& & \\
Executive function & 0.001 & 0.06 \\
Exec func & -0.000 & -0.13 \\
Memory & -0.000 & -0.04 \\
Memory & 0.000 & 0.14 \\
Female & & \\
Couple & -0.041 & -1.89 \\
Mean of dep var & -0.103 & -3.84 \\
\hline Probit analysis. Age & & \\
\hline
\end{tabular}

Probit analysis. Age dummies also included

Sample: all aged 50-59 with a current private pension: $\mathrm{N}=2,020$

In Table 4.4 we look at a broader measure of financial information and expectations that can be taken as an indicator of more general financial insecurity. All ELSA respondents are asked the chances that, at some point in the future, they will not have enough retirement resources to meet their needs. These per cent changes are reported on a scale of $0 \ldots 100$ where 0 means there is no chance at all, and 100 means the respondent is absolutely certain. Using this variable, we construct an indicator taking the value of 1 for those who consider themselves unlikely to have inadequate resources in the future. Table 4.4 shows the results of a similar multivariate analysis to those above which uses this measure of financial insecurity as a dependent variable. Since this question is asked to all respondents it is also an analysis that incorporates all those aged 50-59 regardless of whether they have a private pension, or of the type of private pension they hold. 
Table 4.4 Less than $40 \%$ self reported chance of inadequate resources

\begin{tabular}{l|cr}
\hline & \multicolumn{2}{|c}{$\begin{array}{c}\text { 'enough' resources } \\
\text { Mar. eff }\end{array}$} \\
\hline Group II & 0.051 & 1.59 \\
Group III & 0.056 & 1.62 \\
Group IV & 0.150 & 3.88 \\
& & \\
Medium education & 0.023 & 1.07 \\
High education & 0.036 & 1.66 \\
& & \\
F wealth quintile 2 & 0.150 & 5.51 \\
F wealth quintile 3 & 0.167 & 6.08 \\
F wealth quintile 4 & 0.231 & 8.47 \\
F wealth quintile 5 & 0.321 & 11.71 \\
& & \\
Executive function & 0.137 & 1.72 \\
Exec func & \\
Memory & -0.000 & -1.32 \\
Memory & 0.006 & 0.67 \\
Female & -0.000 & -0.32 \\
Couple & & -1.87 \\
Mean of dep var: & -0.032 & 3.28 \\
Probit analysis. Age dummies also included \\
Sample: all aged 50-59: N = 4,047
\end{tabular}

Once again we see a gradient by numeracy group - the more numerically able are more likely to be in the group who do not expect to have adequate resources - even conditional on both education and financial wealth, both of which also have positive effects. However, these results should be treated with caution since the question asked does not define future needs explicitly. As a result variation in answers may be due to either variation in perceptions of needs or variation in expectations of pension (or other) wealth at retirement. Nevertheless, the analysis does provide evidence of greater financial insecurity amongst less numerate groups even if such insecurity may not necessarily be associated with an inadequacy of retirement resources. Such perceived insecurity may be important for policy as well as perhaps driving the actual likelihood of inadequate retirement resources or indeed other negative outcomes in retirement

This section has revealed that there is some lack of knowledge about pension plans and that the less numerate groups are indeed less likely to know the important details of their plan than their more numerate counterparts. So what could (or should) 
policy makers do about this? The answer to this question depends on which way the causality works. If owning complicated financial products, gives people the opportunity to learn about those assets then encouraging ownership might help (through programmes such as Savings Gateway (at younger ages) for example). However, the majority of the results in this section are based on knowledge about pensions amongst those who have a pension so there does appear to be a case for improving knowledge and understanding of schemes in order to aid financial planning once people have taken out a plan.

If higher numeracy directly improves understanding about financial products, in the long run improving the general level of numeracy in the population would be the most direct way of helping people understand financial products and the implications of any financial decisions that they make. ${ }^{7}$ In the short-run, improving numeracy may be difficult but it may be that providers of financial products have an important role to play. One important way (and probably the most important way in which individuals find out about the financial products that they own is through the provider of those products. Are some providers better than others at presenting and providing information in a way that individuals find easy to understand? And are some types of plans better at conveying key information to less numerate individuals than others? Are there lessons to be learnt from these providers that will help others to improve the way in which they provide information to their customers?

In the final table we develop this idea albeit briefly by using the information on the detailed type of pension plans that individuals have as an additional explanatory variable when looking the extent of individuals' pension knowledge. Although the ELSA questionnaire actually collects information on the precise name of the pension provider, our use of such information is limited by the small numbers of observations we have in any single pension scheme and also (as a natural corollary) the potential disclosiveness of the resulting data. Instead we therefore simply split individuals according to whether they have a public sector pension or not (where public sector pensions are defined as either local authority, teachers, university, NHS, civil service, armed forces or police). In principle, one might expect members of such schemes to be more aware of their

\footnotetext{
${ }^{7}$ Of course from whom people obtain their financial information is also an important issue but this issue is not covered in the ELSA questionnaire.
} 
retirement arrangements since, conditional on earnings, they are typically more generous than non-public sector schemes. Second, public sector schemes are more homogeneous and, combined with the higher unionisation rates in the sector one might expect more straightforward provision of information about of pension arrangements and incentives. Finally, public sector workers have, on average, longer tenures and hence longer to learn about the nature of their pension scheme. ${ }^{8}$

Table 4.5 shows just the coefficients on the public sector variables from a regression where the complete set of covariates (i.e. as in tables 4.1 to 4.4 above) are also included. That is, we control for numeracy, education, other cognitive function, wealth, gender and marital status, and then look at the effect of whether someone is in a public scheme on the same set of outcomes as in our previous tables. Each row of this table provides the relevant coefficients from a probit model where the dependent variable is given by the variable listed in the first column of that row. Initially, in the top panel of Table 4.5, we present the coefficients from a model which just allows a constant 'publicsector' effect. In the lower panel, we present the estimates from a model where we allow this public sector effect to be different for each of our four numeracy groups to see if the public sector schemes are better for some groups than for others.

The results of this exercise are certainly not definitive one way or the other. It is not the case, for example, that public sector workers are universally better informed about their pension arrangements. On average, those in public sector schemes are more likely, other things equal, to know their accrual rate, but less likely to say that they feel that have enough information about their retirement. Looking within numeracy groups it is hard to identify effects that are statistically significant. For example, the positive public sector effect on knowledge of accrual rates is revealed to be strongest amongst the most highly numerate but distinguishing one group from another in terms of statistical significance is difficult. On the other hand the lack of an average effect of public sector pensions on knowledge of expected retirement income is revealed to be due to there being no difference for the higher numeracy groups and a negative but significant effect for the (presumably small) least numerate group.

\footnotetext{
${ }^{8}$ Incidentally, looking at the co-variation of the scheme type with the accrual rate (for those who know it), given that we know the accrual rate for the vast majority of public sector schemes, suggests that the ELSA data does indeed have a good measure of what type of scheme the individual is in.
} 
Table 4.5

Variation in pension knowledge by scheme type, individuals aged 50-59

\begin{tabular}{|c|c|c|c|c|c|c|c|c|}
\hline \multirow[b]{2}{*}{ Dependent variable: } & \multicolumn{2}{|c|}{$\begin{array}{c}\text { Public sector } \\
\text { scheme }\end{array}$} & \multicolumn{2}{|c|}{$\begin{array}{l}\text { Public sector } \\
\text { and numeracy } \\
\text { group II }\end{array}$} & \multicolumn{2}{|c|}{$\begin{array}{l}\text { Public sector } \\
\text { and numeracy } \\
\text { group III }\end{array}$} & \multicolumn{2}{|c|}{$\begin{array}{l}\text { Public sector } \\
\text { and numeracy } \\
\text { group IV }\end{array}$} \\
\hline & $\begin{array}{l}\text { Marg. } \\
\text { effect }\end{array}$ & $\mathrm{z}$ & $\begin{array}{l}\text { Marg. } \\
\text { effect }\end{array}$ & z & $\begin{array}{l}\text { Marg. } \\
\text { effect }\end{array}$ & z & $\begin{array}{l}\text { Marg. } \\
\text { effect }\end{array}$ & $\mathrm{z}$ \\
\hline knows accrual rate & 0.124 & 3.20 & & & & & & \\
\hline knows pension income & -0.008 & -0.27 & & & & & & \\
\hline any answer to indexation & 0.010 & 0.31 & & & & & & \\
\hline not indexed by $>$ prices & -0.009 & -0.23 & & & & & & \\
\hline 'enough' information & -0.076 & -2.82 & & & & & & \\
\hline 'enough' resources & 0.060 & 1.98 & & & & & & \\
\hline knows accrual rate & -0.170 & -1.87 & 0.317 & 1.74 & 0.273 & 1.44 & 0.230 & 1.19 \\
\hline knows pension income & -0.386 & -2.58 & 0.316 & 2.63 & 0.328 & 2.59 & 0.268 & 2.12 \\
\hline any answer to indexation & 0.224 & 1.39 & -0.195 & -1.05 & -0.241 & -1.33 & -0.314 & -1.61 \\
\hline not indexed by $>$ prices & 0.287 & 1.32 & -0.251 & -1.13 & -0.319 & -1.48 & -0.301 & -1.40 \\
\hline 'enough' information & -0.068 & -0.63 & -0.034 & -0.29 & -0.009 & -0.08 & 0.035 & 0.30 \\
\hline 'enough' resources & 0.063 & 0.50 & 0.044 & 0.33 & 0.006 & 0.05 & 0.097 & -0.69 \\
\hline
\end{tabular}

\section{Conclusions}

As the UK has moved more towards a system of individual provision for retirement income, the importance of an individual or household's abilities to take the right choices when it comes to providing for their retirement - either in terms of the decision to accumulate financial wealth, the form in which such wealth should be accumulated, or the decision of when to retire and how that might affect ones retirement income - has increased. In addition to preferences, key components to individual's choices are the information the individual has about the relevant options available and their ability to process this information. Indeed the government's 'informed choice' agenda has explicitly targeted improvements in these latter two dimensions as a goal for government policy.

This paper has looked at data on these issues which is available in England for the first time, and has shown that there is still some way to go with regard to numeracy, financial planning abilities and information about pension saving. We have documented 
systematic differences in numerical abilities of the older population in England and shown that these numeracy differences are strongly positively correlated with a number of measures of retirement information and pension arrangements, even when one takes account of other differences between these individuals in terms of their education, broader cognitive abilities, financial wealth, age, gender and marital status.

The lessons of our analysis are threefold, even though at this point we have only cross-sectional data available on which to base our analysis. Firstly, it shows yet another dimension in which inequalities amongst older individuals are apparent, and in which we see that those at the bottom of the distribution with respect to one measure are often at the bottom of the distribution with respect to other measures. Second, the analysis suggests that in the short run there may be a role for targeting simple retirement planning information at low numeracy, low wealth, low education groups. Third, it suggests that a longer run policy goal might want to target numeracy levels more generally in order to reduce the fraction of the population with low basic skills. Whether such a policy would have knock on effects on to retirement planning arrangements is a more difficult question to answer on the basis of the conditional correlations we have presented here. On this topic in particular, there is much further work to be done. Since the English Longitudinal Study of Ageing is a panel study, new insights will become available as we get the ability to follow the retirement and wealth trajectories of individuals as they age, with different groups defined according to differences in initial cognitive and numerical abilities as well as according to differences in wealth and education levels. The analysis of such data, possibly combined with a behavioural model of individual financial decision making, should be seen as a priority for future research. 


\section{References}

DWP (2003) 'Simplicity, security and choice: working and saving for retirement. Action on occupational pensions', London: The Stationary Office

Lusardi, A. and O. Mitchell, 2005, 'Financial Literacy and Planning: Implications for retirement wellbeing', mimeo, Dartmouth College

Marmot, M., J. Banks, R. Blundell, C. Lessof and J. Nazroo (eds.), 2003, Health, wealth and lifestyles of the older population in England: The 2002 English Longitudinal Study of Ageing, London: IFS

Steel, N., F. Huppert, B. McWilliams and D. Melzer, 2003, 'Physical and cognitive function', in Marmot et al (2003).

Pickering, A., 2002, A simpler way to better pensions, London: Department for Work and Pensions

Sandler, R., 2002, Medium and long term retail savings in the UK, London: The Stationery Office 